\title{
Comparative proteomic analysis of heat stress proteins associated with rat sperm maturation
}

\author{
XIAOMEI WANG ${ }^{1,2^{*}}$, FUJUN LIU $^{1 *}$, XIN GAO $^{3}$, XIN LIU $^{1}$, \\ XIAOJUN KONG ${ }^{1,2}$, HAIYAN WANG ${ }^{1}$ and JIANYUAN LI ${ }^{1,4}$ \\ ${ }^{1}$ Central Laboratory, Yantai Yu Huang Ding Hospital, Yantai, Shandong 264000; ${ }^{2}$ Department of Clinical Laboratory, \\ Clinical Medical College, Ningxia Medical University, Yinchuan, Ningxia 750004; ${ }^{3}$ College of Life Science, Yantai University, \\ Yantai, Shandong 264005; ${ }^{4}$ National Research Institute for Family Planning, Beijing 100081, P.R. China
}

Received May 22, 2015; Accepted January 13, 2016

DOI: $10.3892 / \mathrm{mmr} .2016 .4958$

\begin{abstract}
Heat stress is demonstrated to have an effect on the function of the male testis, however, limited information has been reported on its effects on sperm maturation. In the present study, a comparative proteomic analysis was performed on the rat caput epididymal fluids responsible for sperm maturation, to identify key heat-stress-associated sperm maturation proteins. The results demonstrated 21 proteins corresponding to 29 differential protein spots, including 10 downregulated and 11 upregulated proteins in the heat treatment group. Functional analysis demonstrated that these proteins were primarily involved in enriched reproduction and antioxidant activity. Analysis of western blot and immunohistochemical analysis demonstrated that the expression of antioxidant proteins peroxiredoxin 6 and clusterin were downregulated, and the expression of superoxide dismutase upregulated, in the heat treatment group. Morphological and TUNEL experiments demonstrated that altered nucleus activity occurred in the caput epididymis. The study provided, to the best of our knowledge, novel information for studies on the biological functions of the epididymis and sperm maturation.
\end{abstract}

\section{Introduction}

Heat stress may affect male reproductive functions. The mammalian scrotal temperature is $2-8^{\circ} \mathrm{C}$ lower than the core body temperature (1), and higher temperatures may eliminate the spermatogonial germ cells in the seminiferous tubules, resulting in decreased sperm density $(2,3)$.

Correspondence to: Professor Jianyuan Li, Central Laboratory, Yantai Yu Huang Ding Hospital, 20 Yudong Road, Yantai, Shandong 264000, P.R. China

E-mail: sdscli@126.com

${ }^{*}$ Contributed equally

Key words: heat stress, epididymis, microenvironment, proteome, sperm maturation
This may further induce testicular tissue apoptosis and morphological changes affecting sperm production processes, resulting in infertility (4-6). In addition, increased scrotal temperatures resulting from occupational exposure, lifestyle or cryptorchidism are notable factors leading to male infertility (7). Limited research has been conducted on the epididymis (8). Spermatozoa are produced in the testis and acquire the maturation ability in the epididymis, particularly in the caput epididymis, by interacting with the epididymal fluids $(9,10)$. Although numerous studies have investigated the effect of heat stress of the testis on spermatogenesis (11-13), the association of heat stress and sperm maturation has yet to be elucidated.

In the present study, a short heat stress was performed on the rat epididymis to identify the affected sperm maturation proteins. The epididymal fluid proteins identified may provide useful information for the understanding of sperm maturation, and provide clues for the screening of male contraceptive agents.

\section{Materials and methods}

Sample preparation. Male Sprague-Dawley rats $(\mathrm{n}=16$; weight, 300-400 g; age, 8-10 weeks) were provided by the Experimental Animal Center of Binzhou Medical College (Shandong, China). They were housed at $20 \pm 2^{\circ} \mathrm{C}$ with ad libitum access to food and water and a $12 \mathrm{~h}$ light/dark cycle. Rats were randomly divided into two groups and acclimatized to the experimental conditions for one week prior to commencement of the experiments as follows: The normal control group, rats were in the water bath for $60 \mathrm{~min}$ once prior to the experiment at room temperature; the heat treatment group, rats received heat shock pretreatment in a water bath once prior to the experiment at $42^{\circ} \mathrm{C}$ for $60 \mathrm{~min}$. The animals were then sacrificed by intraperitoneal injection with ketamine (2.4 ml.kg; Fujian Gutian Yuanhang Medical Company, Ningde, China). The study was approved by the ethics committee of Yu Huang Ding Hospital (Yantai, China).

Protein extraction. Rat caput epididymides were collected for fluid protein extraction. Briefly, the epididymides were coarsely minced and gentle pressure was applied 
to release luminal fluid into phosphate-buffered saline. Following centrifugation at $5,700 \mathrm{x} \mathrm{g}$ at $4^{\circ} \mathrm{C}$ for $10 \mathrm{~min}$ and microscopic examination (DM LB2; Leica Microsystems, Wetzlar, Germany), the resultant sperm-free supernatant was precipitated with four volumes of ice-cold acetone. The precipitates were dissolved in $3 \mathrm{ml}$ protein lysis buffer (Sigma-Aldrich, St. Louis, MO, USA) consisting of $7 \mathrm{M}$ urea, $2 \mathrm{M}$ thiourea, 4\% (w/v) 3-[(3-cholamidopropyl) dimethylammonio]-1-propanesulfonate and $65 \mathrm{mM}$ dithiothreitol. Protein concentration was determined using the Bradford assay (Thermo Fisher Scientific, Inc., Waltham, MO, USA) and the samples were stored at $-80^{\circ} \mathrm{C}$.

Two-dimensional gel electrophoresis $(2-D E)$. The first dimension of the 2-DE was the separation of proteins by isoelectric focusing using $18 \mathrm{~cm}$ nonlinear $\mathrm{pH}$ 3-10 immobilized $\mathrm{pH}$ gradient (IPG) strips (GE Healthcare Life Sciences, Chalfont, UK). Subsequent to reduction and iodoacetamide (Sigma-Aldrich), the IPG strips were run on a $12.5 \%(w / v)$ SDS-PAGE. The gels were stained with Coomassie Brilliant Blue R-350 (GE Healthcare Life Sciences), and were scanned by the Z320 scanner (Founder Technology Group Co., Ltd., Beijing, China). The gel maps were analyzed with Imagemaster 2D Platinum software, version 6.0 (GE Healthcare Life Sciences), and the electrophoresis experiments were performed in triplicates. The gel spots were excised, destained with $25 \mathrm{mM} \mathrm{NH} \mathrm{mCO}_{3} / 50 \%$ (v/v) acetonitrile (both purchased from Yantai Sanhe Chemical Reagent Company, Yanta, China), and digested by trypsin (Thermo Fisher Scientific, Inc.) in $25 \mathrm{mM} \mathrm{NH}_{4} \mathrm{HCO}_{3}$ at $37^{\circ} \mathrm{C}$ for $12 \mathrm{~h}$. The resulting peptides were analyzed using the Applied Biosystems Voyager-DE STR Biospectrometry Workstation (Thermo Fisher Scientific, Inc.). The spectra data of the mass spectrometry were searched against the NCBInr database for Rattus norvegicus with Mascot (www.matrixscience.com/; Matrix Science Ltd., London, UK). Providing that the protein score was $>60$ and the matched peptides $\geq 4$ in the peptide mass fingerprinting search, the protein was confirmed as a successful identification. In the case that one gel spot corresponded to $>1$ protein, then the protein with the highest score was selected.

Bioinformatics. Proteins were distinguished functionally by a step-by-step classification and each protein was placed in only one category. Broad functions were classified according to the annotations in the PIR database (pir.georgetown.edu) and gene ontology (GO) annotations (www.geneontology.org), including molecular function and biological process.

Immunohistochemical analysis. The rat caput epididymides were fixed in Bouin's solution (Sigma-Aldrich) for $10 \mathrm{~h}$ at room temperature, and embedded with paraffin (Yantai Sanhe Chemical Reagent Company). For antigen retrieval, the $4-\mu \mathrm{m}$ thick sections were cut and heated in a microwave oven for $15 \mathrm{~min}$. To eliminate the endogenous peroxidases, $3 \%(\mathrm{v} / \mathrm{v})$ $\mathrm{H}_{2} \mathrm{O}_{2}$ was used to incubate the sections for $10 \mathrm{~min}$. Subsequent to antigen retrieval, blocking was conducted with $3 \%$ bovine serum albumin for $30 \mathrm{~min}$ at $37^{\circ} \mathrm{C}$. Then, the samples were incubated with the following antibodies (dilution, 1:50) overnight at $4^{\circ} \mathrm{C}$ : Polyclonal anti-rabbit clusterin (sc-8354), peroxiredoxin 6 (sc-134478) and superoxide dismutase-1 (sc-11407) (all purchased from Santa Cruz Biotechnology,
Inc., Dallas, TX, USA). Sections were washed three times with Tris-buffered saline (TBS) and then incubated with horseradish peroxidase (HRP)-conjugated anti-rabbit IgG (dilution, 1:500; Zhongshan Beijing Biotechnology Co., Ltd., Guangdong, China) for $1 \mathrm{~h}$ at $37^{\circ} \mathrm{C}$. A DAB kit (Zhongshan Beijing Biotechnology Co., Ltd.) was used to reveal the binding sites, and then the sections were counterstained with hematoxylin and eosin (HE; Abcam, Cambridge, MA, USA), and mounted for bright-field microscopy with a DM LB2 microscope (Leica Microsystems). Pre-immune rabbit IgG (sc-2027; Santa Cruz Biotechnology, Inc.) was used instead of the primary antibody as negative control.

Western blot analysis. Protein samples $(50 \mu \mathrm{g})$ were separated by $12.5 \%(\mathrm{w} / \mathrm{v}) \mathrm{SDS}-\mathrm{PAGE}$, and transferred to polyvinylidene fluoride membranes. Subsequently, the membranes were blocked with $5 \%(\mathrm{w} / \mathrm{v})$ skimmed milk for $1 \mathrm{~h}$ and incubated with clusterin, peroxiredoxin 6 and superoxide dismutase-1 primary antibodies at room temperature for $1 \mathrm{~h}$. Membranes were washed three times with TBS-Tween 20 and incubated with an HRP-conjugated anti-IgG for $1 \mathrm{~h}$. A DAB kit (Zhongshan Beijing Biotechnology Co., Ltd.) was used to visualize the proteins. Western blot images were analyzed by densitometric scanning and quantified using ImageQuant TL software (version 7.0; GE Healthcare).

Tunel assay. DNA fragmentation was visualized by TUNEL assay using the In Situ Cell Death Detection Kit, Fluorescein (Roche Diagnostics GmbH, Mannheim, Germany), according to the manufacturer's instructions. Sections were photographed by confocal laser scanning microscopy using a LSM-510 META device (Carl Zeiss AG, Oberkochen, Germany). The TUNEL positive cells (green staining) were photographed and evaluated qualitatively using ImageQuant TL 7.0 software (GE Healthcare). Negative controls were processed with TUNEL-Label Solution (Roche Diagnostics) without terminal transferase instead of the TUNEL reaction mixture.

Statistical analysis. The unpaired t-test was used to assess the differences among groups, using SPSS software, version 13.0 (SPSS, Inc., Chicago, IL, USA). Data are presented as the mean \pm standard deviation. $\mathrm{P}<0.05$ was considered to indicate a statistically significant difference.

\section{Results}

Morphological characteristics of rat caput epididymis treated by short-term heat treatment. As demonstrated in Fig. 1, HE staining of rat caput epididymis indicated no significant changes in cellular morphologies or lumen dimensions in the caput epididymides of the heat treatment group compared with the normal control group. However, nuclei cavitations were observed in the rat caput epididymis of the heat treatment group (Fig. 1; HE staining).

Further cellular localizations of clusterin (Clu), peroxiredoxin 6 (Prdx6) and superoxide dismutase (Sod1) were investigated by immunohistochemistry. The results demonstrated that Sod1, Prdx6 and Clu were mainly expressed by epididymal epithelial cells (Fig. 1). Following 

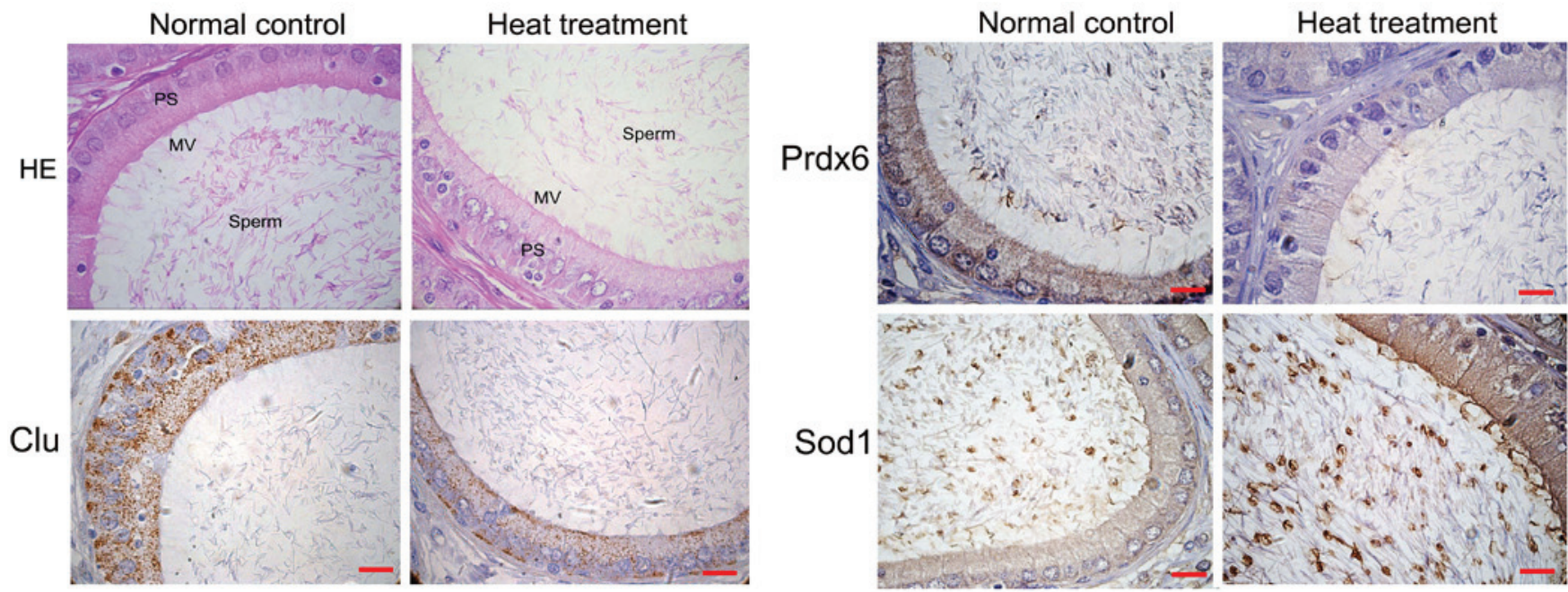

Figure 1. Morphological and immunohistochemical analysis of caput epididymis in normal control and heat-treated rats. Scale bar, $40 \mu \mathrm{m}$. HE, hematoxylin and eosin; PS, principle cells; MV, microvilli. Clu, clusterin; Prdx6, peroxiredoxin 6; Sod1, superoxide dismutase.

heat treatment, Prdx6 and Clu demonstrated decreased levels of expression, and Sod1 indicated increased levels compared with the normal control group (Fig. 1).

Identification of differentially expressed proteins in rat caput epididymis with short-term heat stress. 2-DE followed by mass identification was performed to identify differentially expressed proteins. Triple gels were repeatedly made and statistically compared by Imagemaster software. A total of 29 protein spots demonstrated different density levels between the heat treated and normal control groups (fold change, $>1.5$ ). From the total number of spots, 21 unique proteins were identified, including 11 proteins upregulated and 10 proteins downregulated in the treatment group (Fig. 2; Table I).

Broad functional analysis. All differentially expressed proteins were broadly classified into seven functional groups according to GO and literature annotations (Table I). These proteins were mainly involved in functions of structure (23.8\%), reproduction (19\%), antioxidants (19\%), metabolism, chaperones, proteases/inhibitors and transporters (Table I).

Western blot analysis. Antioxidant-associated (Sod1 and Prdx6) and a reproduction-associated protein (Clu) were selected for validation of their expression using western blotting. As demonstrated in Fig. 3, the heat treatment group exhibited significantly lower expression levels of Prdx6 and Clu compared with the normal control group $(\mathrm{P}<0.05$; Fig. 3$)$. In addition, higher expression levels of Sod1 were observed in the heat-treated group compared with the normal control group $(\mathrm{P}<0.05$; Fig. 3$)$. The results were consistent with the proteomic analysis.

TUNEL assay. TUNEL assay was performed to determine the effect of heat stress on the apoptotic status. Positive stained cells in the heat-treated epididymis $(68 \pm 5.2 \%)$ was significantly higher than that in the control group $(16 \pm 4.2 \%)$. As demonstrated in Fig. 4, positive staining results were observed in epididymal epithelial cells from the heat-treated rats.

\section{Discussion}

Increased temperature has an effect on the male reproductive ability by declining male fertility (14). Heat stress may have a negative effect on male fertility through oxidant damage by producing ROS and further changing the intracellular signal transduction (15). This effect may induce germ cell loss and poor semen quality, however, its effect on sperm maturation, particularly on the sperm maturation milieu, is not clear $(16,17)$. In the present study, a short-term heat stress was performed on rats by exposure to high environmental temperature $\left(42^{\circ} \mathrm{C}\right)$ for $1 \mathrm{~h}$, followed by identification of altered key sperm milieu proteins by comparing a proteomic analysis of caput epididymal fluids. To the best of our knowledge, this is the first study attempting to identify sperm milieu proteins affected by short-term heat stress, and may provide information for further understanding of sperm maturation.

Caput epididymal fluids provide a microenvironment for sperm maturation for active epithelial secretion and absorption activity (18). This microenvironment serves as the first step for the sperm to obtain its maturation ability. The present study hypothesized that heat stress may affect caput epididymal epithelial protein expression patterns, further altering caput epididymal fluid proteins that are mainly associated with sperm maturation.

The present study may provide understanding for heat-stress-induced reproductive dysfunction in males. The morphological analysis of the present study demonstrated no significant differences between the treated and normal control groups of rat caput epididymides. This implies that short-term heat stress may not affect epithelial structures. To further explore the effect on the main activities of caput epididymis on protein synthesis and secretion, a comparative proteomic study was performed to identify differentially expressed fluid proteins. A total of 29 proteins were identified to have varying expression levels between the treated and 
Table I. Differentially expressed proteins between normal control and heat treatment of rat caput epididymis.

\begin{tabular}{|c|c|c|c|c|c|c|}
\hline Spot no. & $\begin{array}{l}\text { Swiss-Prot } \\
\text { accession }\end{array}$ & $\begin{array}{c}\text { Molecular } \\
\text { weight }\end{array}$ & $\begin{array}{l}\text { Gene } \\
\text { name }\end{array}$ & Score & Protein name & $\begin{array}{l}\text { Treatment } \\
\text { /control }\end{array}$ \\
\hline \multicolumn{7}{|c|}{ Antioxidant } \\
\hline F11 & O35244 & 24860 & $\operatorname{Prdx} 6$ & 835 & Peroxiredoxin-6 & 0.38 \\
\hline A4 & P07632 & 16073 & Sod1 & 397 & Superoxide dismutase $(\mathrm{Cu}-\mathrm{Zn})$ & 2.15 \\
\hline G3 & P09606 & 42982 & Glul & 853 & Glutamine synthetase & 0.43 \\
\hline G16 & P11232 & 12008 & Txn & 294 & Thioredoxin & 2.32 \\
\hline \multicolumn{7}{|c|}{ Chaperone } \\
\hline A1 & B0BNA5 & 16036 & Cotl1 & 73 & Coactosin-like protein & 2.54 \\
\hline \multicolumn{7}{|c|}{ Metabolism } \\
\hline $\mathrm{C} 12$ & O88989 & 36631 & Mdh1 & 82 & Malate dehydrogenase, cytoplasmic & 0.33 \\
\hline H6 & P19804 & 17386 & Nme2 & 553 & Nucleoside diphosphate kinase B & 0.41 \\
\hline A20 & P47819 & 49984 & Gfap & 91 & Glial fibrillary acidic protein & 2.65 \\
\hline \multicolumn{7}{|c|}{$\mathrm{P} / \mathrm{P}$ inhibitor } \\
\hline A17 & P01041 & 11303 & Cstb & 106 & Cystatin-B & 3.01 \\
\hline G6 & Q5XI73 & 23450 & Arhgdia & 181 & Rho GDP-dissociation inhibitor 1 & 0.36 \\
\hline \multicolumn{7}{|c|}{ Reproduction } \\
\hline $\mathrm{F} 2$ & P06911 & 20828 & Lcn5 & 849 & Epididymal-specific lipocalin-5 & 2.89 \\
\hline A15 & P12020 & 28741 & Crisp1 & 459 & Cysteine-rich secretory protein 1 & 2.64 \\
\hline F13 & P05371 & 51970 & Clu & 430 & Clusterin & 0.28 \\
\hline $\mathrm{H} 2$ & P30904 & 12640 & Mif & 127 & Macrophage migration inhibitory factor & 0.34 \\
\hline \multicolumn{7}{|l|}{ Structure } \\
\hline $\mathrm{F} 24$ & P60711 & 42052 & Actb & 599 & Actin, cytoplasmic 1 & 3.12 \\
\hline E2 & P62986 & 8560 & Uba52 & 331 & Ubiquitin & 0.27 \\
\hline F19 & P63259 & 42108 & Actg1 & 482 & Actin, cytoplasmic 2 & 2.57 \\
\hline E13 & Q63544 & 12969 & Sncg & 140 & $\gamma$-Synuclein & 0.38 \\
\hline B8 & Q6P9T8 & 50225 & Tubb4b & 425 & Tubulin $\beta-4 B$ chain & 2.36 \\
\hline \multicolumn{7}{|c|}{ Transporter } \\
\hline G24 & P02767 & 15824 & $\operatorname{Ttr}$ & 329 & Transthyretin & 0.47 \\
\hline F8 & P31044 & 20902 & Pebp1 & 823 & Phosphatidylethanolamine-binding protein 1 & 2.63 \\
\hline
\end{tabular}

$\mathrm{P} / \mathrm{P}$ inhibitor, protease/protease inhibitor.

normal control groups. A broad functional classification categorized these proteins into seven functional groups, and the majority were associated with antioxidant and reproductive functions.

Antioxidant enzymes in the epididymal lumen protect spermatozoa from oxidative damage. Optimal antioxidant levels serve crucial roles in sperm function (particularly sperm motility) (19). The results of the present study demonstrated that Prdx6 and Glul, two proteins with protective roles in sperm, had lower expression levels in the epididymal fluids following heat stress, suggesting that an altered protection balance may occur in epididymal fluids induced by heat stress. Another two ROS-associated proteins, Sod1 and Txn, presented with upregulated expression levels, potentially implicating an epididymis response partly compensating for spermatozoa oxidative damage following heat stress. These proteins were associated with certain aspects of sperm maturation (20), and the association of their function with heat stress was further investigated. Another promising function group was the reproduction-associated proteins. These proteins were established sperm proteins, such as $\mathrm{Clu}$, a heterodimeric glycoprotein produced by a variety of tissues and present in numerous biological fluids (21). In addition, Clu is a major component of epididymal fluids, and mainly secreted in the caput epididymis. Previous studies have demonstrated that $\mathrm{Clu}$ is involved in cell adhesion, apoptosis and transformation functions, and sperm maturation $(18,22,23)$. In the present study, Clu levels were observed to be significantly lower following heat stress, suggesting that heat stress may have a direct effect on the epididymal fluid component, and directly or indirectly alter sperm maturation. The above proteins should be further validated and investigated to identify their regulation and roles in sperm maturation.

In the present study, western blot analysis validated the expression levels of proteins in epididymal fluids, and immunohistochemical analysis identified the expression of proteins in the epididymal epithelia. The results indicated 


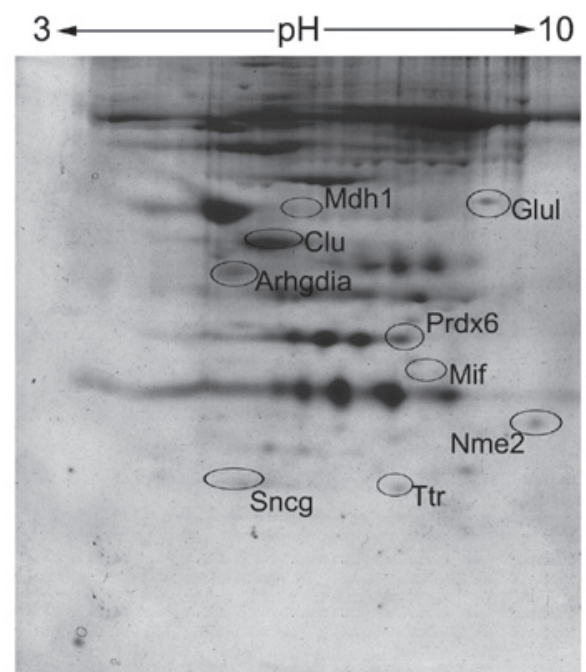

Normal control

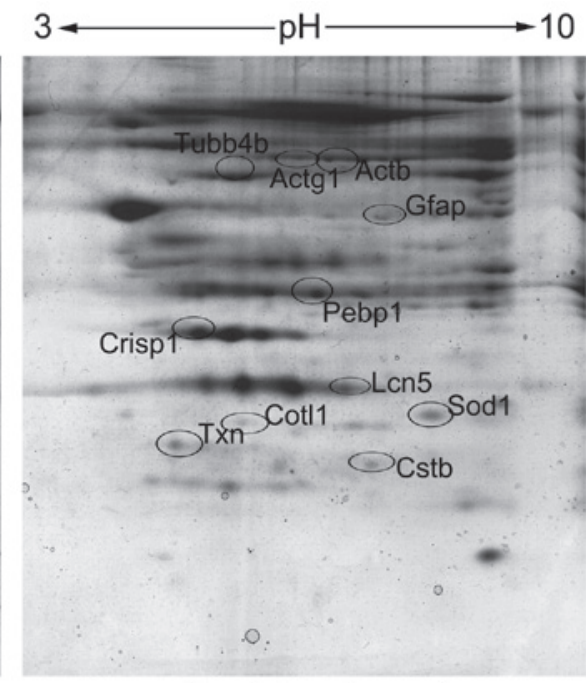

Heat treatment

Figure 2. Separation and identification of differentially expressed rat caput epididymal fluid proteins by $2 \mathrm{D}$ gel electrophoresis.
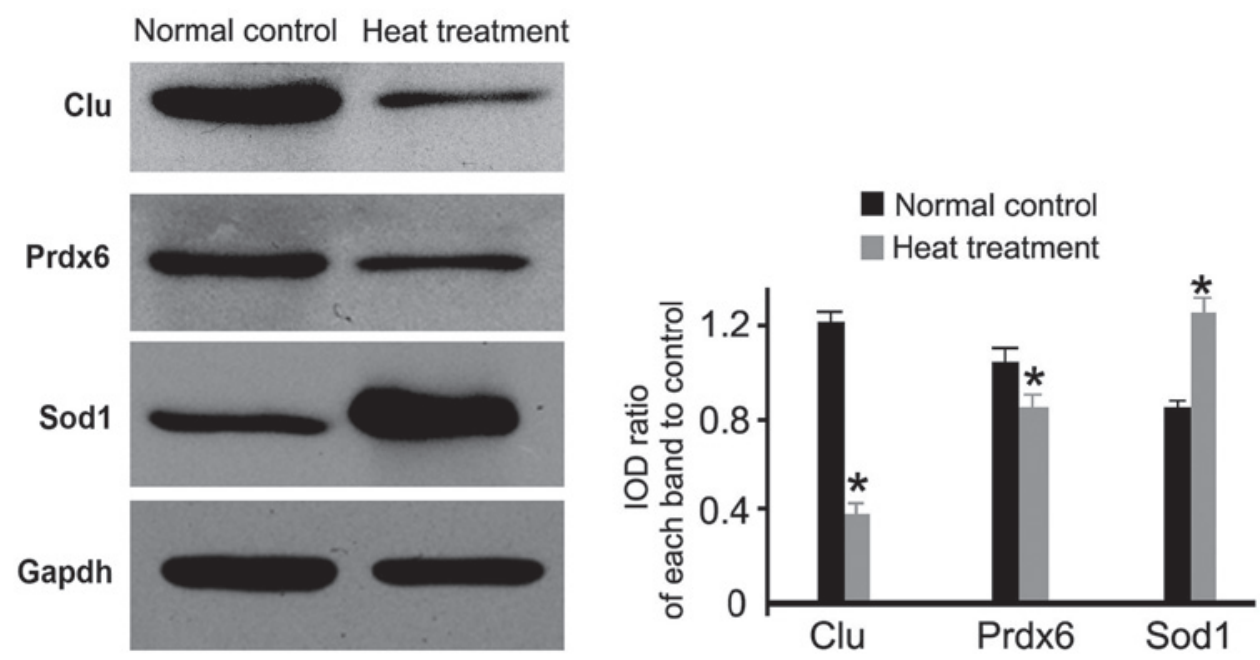

Figure 3. Western blot analysis of Clu, Prdx6 and Sod1 protein expression levels in normal control and heat treatment rat caput epididymal fluids. *P<0.05 vs. the normal control. Clu, clusterin; Prdx6, peroxiredoxin 6; Sod1, superoxide dismutase; IOD, integrated optical density.
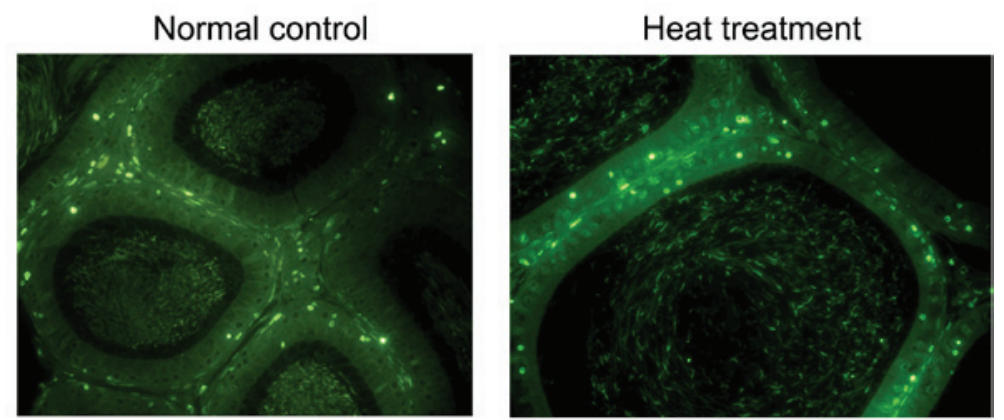

Figure 4. Heat induced apoptosis of control and heat-treated rat epididymides detected by TUNEL assay (magnification, x400).

the same altered expression in epididymal epithelia and fluids, suggesting that heat stress induced protein expression changes in epididymal fluids.

In conclusion, by utilizing heat stress exposure on rat epididymis, a set of differentially expressed caput epididymal fluid proteins were identified, whose activities were associated with sperm maturation. The results of the present study, in combination with data from our previous work, will be used as the basis for future studies on biological functions of the epididymis and sperm maturation. 


\section{Acknowledgements}

The current study was supported by the National Natural Science Foundation of China (grant no. 81300533) and Shandong Provincial Natural Science Foundation, China (grant nos. ZR2013HQ002 and ZR2014HQ068).

\section{References}

1. Banks S, King SA, Irvine DS and Saunders PT: Impact of a mild scrotal heat stress on DNA integrity in murine spermatozoa. Reproduction 129: 505-514, 2005.

2. Wang P, Li Y, Hu XC, Cai XL, Hou LP, Wang YF, Hu JH, Li QW, Suo LJ, Fan ZG and Zhang B: Cryoprotective effects of low-density lipoproteins, trehalose and soybean lecithin on murine spermatogonial stem cells. Zygote 22: 158-163, 2014.

3. Rasooli A, Taha Jalali M, Nouri M, Mohammadian B and Barati F: Effects of chronic heat stress on testicular structures serum testosterone and cortisol concentrations in developing lambs. Anim Reprod Sci 117: 55-59, 2010.

4. Li YS, Piao YG, Nagaoka K, Watanabe G, Taya K and Li CM: Preventive effect of tert-butylhydroquinone on scrotal heat-induced damage in mouse testes. Genet Mol Res 12 5433-5441, 2013

5. Pei Y, Wu Y, Cao J and Qin Y: Effects of chronic heat stress on the reproductive capacity of male Rex rabbits. Livest Sci 146: 13-21, 2012.

6. Takahashi M: Heat stress on reproductive function and fertility in mammals. Reprod Med Biol 11: 37-47, 2012.

7. Kim B, Park K and Rhee K: Heat stress response of male germ cells. Cell Mol Life Sci 70: 2623-2636, 2013.

8. Hou Y, Wang X, Lei Z, Ping J, Liu J, Ma Z, Zhang Z, Jia C, Jin M, Li X, et al: Heat-stress-induced metabolic changes and altered male reproductive function. J Proteome Res 14: 1495-503, 2015.

9. Downs SM: Nutrient pathways regulating the nuclear maturation of mammalian oocytes. Reprod Fertil Dev: Mar 24, 2015 (Epub ahead of print).

10. Gatti JL, Castella S, Dacheux F, Ecroyd H, Métayer S, Thimon V and Dacheux JL: Post-testicular sperm environment and fertility. Anim Reprod Sci 82-83: 321-339, 2004.
11. Shen H, Liao K, Zhang W, Wu H, Shen B and Xu Z: Differential expression of peroxiredoxin 6 , annexin A5 and ubiquitin carboxyl-terminal hydrolase isozyme L1 in testis of rat fetuses after maternal exposure to di-n-butyl phthalate. Reprod Toxicol 39: 76-84, 2013

12. Fu-Jun L and Xiao-Fang S: Comparative analysis of human reproductive proteomes identifies candidate proteins of sperm maturation. Mol Biol Rep 39: 10257-10263, 2012.

13. Xun W, Shi L, Cao T, Zhao C, Yu P, Wang D, Hou G and Zhou H: Dual functions in response to heat stress and spermatogenesis: Characterization of expression profile of small heat shock proteins 9 and 10 in goat testis. Biomed Res Int 2015: 686239, 2015.

14. Paul C, Teng S and Saunders PT: A single, mild, transient scrotal heat stress causes hypoxia and oxidative stress in mouse testes, which induces germ cell death. Biol Reprod 80: 913-919, 2009.

15. Sanocka D and Kurpisz M: Reactive oxygen species and sperm cells. Reprod Biol Endocrinol 2: 12, 2004.

16. Saez F, Frenette G and Sullivan R: Epididymosomes and prostasomes: Their roles in posttesticular maturation of the sperm cells. J Androl 24: 149-154, 2003.

17. Dacheux JL and Dacheux F: New insights into epididymal function in relation to sperm maturation. Reproduction 147: R27-R42, 2014.

18. Maldera JA, Vasen G, Ernesto JI, Weigel-Muñoz M, Cohen DJ and Cuasnicu PS: Evidence for the involvement of zinc in the association of CRISP1 with rat sperm during epididymal maturation. Biol Reprod 85: 503-510, 2011.

19. Park K, Jeon S, Song YJ and Yi LS: Proteomic analysis of boar spermatozoa and quantity changes of superoxide dismutase 1 , glutathione peroxidase, and peroxiredoxin 5 during epididymal maturation. Anim Reprod Sci 135: 53-61, 2012.

20. DacheuxJL,BelleannéeC,GuyonnetB,Labas V,Teixeira-Gomes AP, Ecroyd H, Druart X, Gatti JL and Dacheux F: The contribution of proteomics to understanding epididymal maturation of mammalian spermatozoa. Syst Biol Reprod Med 58: 197-210, 2012.

21. Gbormittah FO, Bones J, Hincapie M, Tousi F, Hancock WS and Iliopoulos O: Clusterin glycopeptide variant characterization reveals significant site-specific glycan changes in the plasma of clear cell renal cell carcinoma. J Proteome Res 14: 2425-2436, 2015.

22. Labas V, Spina L, Belleannee C, Teixeira-Gomes AP, Gargaros A, Dacheux F and Dacheux JL: Analysis of epididymal sperm maturation by MALDI profiling and top-down mass spectrometry. J Proteomics 113: 226-243, 2015.

23. Baker MA, Weinberg A, Hetherington L, Villaverde AI and Velkov T: Analysis of protein thiol changes occurring during rat sperm epididymal maturation. Biol Reprod 92: 11, 2015. 